\title{
Traumatic experiences in childhood and the development of psychosis spectrum disorders
}

\author{
Chantal Michel ${ }^{1} \cdot$ Jochen Kindler ${ }^{1} \cdot$ Michael Kaess $^{1,2}$
}

Published online: 19 January 2022

(C) The Author(s), under exclusive licence to Springer-Verlag GmbH Germany 2022

Childhood trauma is defined as a frightening, dangerous, or violent event that poses a threat to a child's life or bodily integrity which can capture a range of severe adverse experiences, such as physical, sexual and emotional abuse, neglect, parental death, and bullying, affecting about one-third of the general population [1]. Exposure to childhood trauma is common in patients with early psychosis and also those with clinical high risk (CHR) for psychosis, with up to $80 \%$ of patients having been exposed to some traumatic experience, and is associated with increased symptomatology $[2,3]$.

Relatedly, there is growing evidence for the association between childhood trauma and the development of psychotic symptoms later in life [3]. Results of a meta-analysis by Pastore et al. [4] published in this issue of European and Adolescent Psychiatry reveal the consequences of childhood trauma: development of psychotic symptoms was not necessarily associated with a specific type of trauma, but rather, with other variables such as age of exposure and especially multi-victimization (e.g., cumulative effect of bullying) that appear to be more strongly related to psychosis risk [4, 5]. Notably, although the cumulative effect (i.e., dose-response effect) of trauma appears to be of highest importance for risk, rather than specific types, those forms of traumas characterised by an intention to harm, such as bullying, and maltreatment by an adult, demonstrate a strong overall risk for psychosis, even more so than the death of a parent [5]. Findings were discussed through the lens of the diathesisstress model, which attempts to explain mental disorders as the development from a genetic/biological predisposition for

Michael Kaess

michael.kaess@upd.ch

1 University Hospital of Child and Adolescent Psychiatry and Psychotherapy, University of Bern, Bolligenstrasse 111, Haus A, 3000 Bern 60, Switzerland

2 Department of Child and Adolescent Psychiatry, Centre for Psychosocial Medicine, University Hospital Heidelberg, Heidelberg, Germany that illness (diathesis) combined with stressful conditions playing a precipitating or facilitating role [1-3]. Consequentially, results of the meta-analysis highlight two important aspects of vulnerability for the individual: (1) the psychological/interpersonal stressors that may encounter throughout lifetime alongside with other environmental factors (i.e., substance abuse, low socioeconomic status), and (2) a genetic/biological predisposition.

Considering interpersonal trauma as a severe stressor, this emphasises the need for early universal or selective prevention programs, including school-based interventions focussing on anti-bullying, and interventions aimed at reducing domestic and family violence, for example, in the effort to buffer against potential psychopathological effects of such trauma. Regarding genetic predisposition, traumatic childhood experiences may not only interact with genetic vulnerability (i.e., first degree relative with psychosis) [5] leading to psychosis, but also other biological factors (e.g., hypothalamic-pituitary-adrenal axis dysregulation, structural brain alterations) as well as psychological mechanisms (e.g., dysfunctional cognitive schemata, affective dysregulation) that might help to explain this link [1,3]. One example of a potential biological component linking psychosis and trauma is the brain-derived neurotropic factor (BDNF), a protein important for neurogenesis, neuroplasticity as well as for the release of neurotransmitters in the brain, particularly within the hippocampus, a brain structure important not only for psychosis, but also related to childhood trauma [3]. BDNF is considered a potential biomarker for psychosis, given that multiple studies have found that low levels of BDNF are associated with psychosis risk [3]. Further, the hippocampus, which might be effected by low BDNF, is an important structure for accurate processing of social memories and therefore is relevant for social cognition. Impairments in social cognition are subjectively perceived as important in adolescents with a CHR state [6]. These impairments might be perceived as stressful for CHR patients and could lead 
to further stress, and in line with the diathesis stress model, increase the risk for the development of psychosis.

Several studies have explored other proposed models explaining the connection between traumatic events and psychotic symptoms [1]. Models that focus on psychological factors highlight the role of dysfunctional cognitive schemata and affective dysregulation as the primary psychological mechanisms. Cognitive models suggest that traumatic events drastically alter core cognitive schemata in children and adolescents, promoting maladaptive beliefs of self, the world, and their future (Beck's cognitive triad) so that the world is perceived as negative and threatening [1]. These distortions of thought significantly influence the interpretation of internal and external experiences, and arguably lay the foundation for persecutory delusions experienced by those struggling with psychosis [1]. Associations between paranoia and negative cognitions about self and others have been well established in clinical and nonclinical samples and it is posited that these cognitive distortions may be one of the primary mediators in the relationship between psychosis and trauma [3]. More specifically, a statistically significant correlation was found between negative attributional styles associated with trauma and positive symptoms of psychosis, most notably, hallucinations [3]. Further, a salient relationship between the content of childhood trauma and the subsequent themes of delusions and hallucinations was reported [3]. Additionally, recent cognitive models suggest an association between negative schematic beliefs and a predisposition to both paranoia and hallucinations, with negative beliefs about self and others being most strongly associated with a predisposition to paranoid thoughts. Therefore, persecutory delusions can be viewed as threat beliefs, which may emerge as a response to the experience of interpersonal stress and trauma [3].

In addition, the link between childhood traumatic events and affect dysregulation might explain the high rates of comorbidity, particularly in disorders with affect dysregulation problems (such as depression, anxiety, and substance use) present in those who experienced childhood trauma with or without psychosis [1,3]. The link between childhood traumatic events and affect dysregulation has been well established and recent research has also proposed an affective model to describe the connection between trauma and psychosis. Emotion regulation is considered a developmental task highly influenced by a safe, emotionally attuned and regulated caregiving environment. Interpersonal trauma, especially traumatic events occurring within the caregiving environment, greatly disrupt the development of adaptive emotion regulation strategies, resulting in increased risk for emotional dysregulation [1,3] and therefore, an increased risk of psychosis.

Overall, childhood trauma increases the risk for psychosis, affects severity and type of psychotic symptoms, frequency of comorbid conditions, including depression and substance use, and is linked to more severe functional impairment in individuals with psychosis $[1,3]$. Psychotic experiences-especially hallucinatory-and trauma, predict persistence of psychosocial problems in adolescents, underscoring the need to assess psychotic experiences and trauma in mental health screening programs [7]. Whereas most screening programs for psychosocial problems in adolescence focus mainly on internalising and externalising problems, the need for additional items concerning psychotic and traumatic experiences is evident [2] and using evidencebased assessments of trauma to help identify their impact is critical. Assessment of psychotic experiences in children and adolescents can be challenging and the need for more in-depth studies into developmental peculiarities in the early detection and intervention of psychoses in youth remains [8]. Crucially, there is a strong need to develop diagnostic tools that consider all aspects of development in children and adolescents, including biological, affective, cognitive, and social characteristics [8].

Carefully considering the impact of trauma on the development of psychosis is not only important in the accurate assessment and diagnosis of psychosis spectrum disorders, but ultimately in treatment as well. The use of cognitive behavioural therapy and other cognitive behavioural interventions in the treatment of traumatized youth, are suggested as potentially beneficial, which include principles of affect recognition, modulation and regulation, cognitive restructuring, and enhancing future safety. However, sufficient evidence on the usefulness of such interventions for children and adolescents with co-occurring trauma and psychosis is still lacking, although emerging evidence in adults is encouraging [3]. Future studies should specifically offer tailored treatments to patients with psychosis spectrum symptoms and a history of childhood trauma aimed at reducing sensitivity to stress in daily life. Therefore, the efficacy of stress reduction strategies including self-relaxation techniques, adaptation of cognitive behavioural therapy to remediate stress sensitivity, and mindfulness-based interventions could be used as add-on therapeutic approaches in psychosis spectrum patients $[1,2,4,9]$. Further, burgeoning research using ecological momentary assessment and ecological momentary interventions presents a promising option for intervention, especially for the monitoring of symptoms and patient engagement in more readily available day-to-day support, not limited to (usually weekly) sessions with a therapist [10]. Other potential applications of such technology include the immediate recognition and a prompt via a digital app to the person challenging their delusional thoughts, for example, helping the patient to engage in cognitive re-structuring at the time of experiencing the delusional thoughts. Additionally, the future of mobile technology in mental health care is growing increasingly prominent as ecological momentary 
assessment and intervention could help to monitor elevated sensitivity and lack of resilience to socio-environmental stress and enhanced threat anticipation in daily life, and therefore might be valuable in empowering people in more effective self-management [10].

In sum, the experiences of childhood trauma present not only a risk factor for the development of, but also the specific manifestation of, psychotic experiences in later life. Future longitudinal research exploring proposed and specific mechanisms would help to differentiate trajectories leading from childhood trauma to distinct lifetime psychopathologies including psychosis spectrum disorders. Moreover, future developments of the treatment strategies (including digital interventions) targeting traumatic experiences and their role in psychosis are needed, and might provide substantial therapeutic benefit to those affected by both trauma and psychosis spectrum disorders.

\section{Declarations}

Conflict of interest The authors declare that they have no conflict of interest.

\section{References}

1. Misiak B, Krefft M, Bielawski T, Moustafa AA, Sąsiadek MM, Frydecka D (2017) Toward a unified theory of childhood trauma and psychosis: a comprehensive review of epidemiological, clini$\mathrm{cal}$, neuropsychological and biological findings. Neurosci Biobehav Rev 75:393-406. https://doi.org/10.1016/j.neubiorev.2017.02. 015

2. Mayo D, Corey S, Kelly LH, Yohannes S, Youngquist AL, Stuart BK, Niendam TA, Loewy RL (2017) The role of trauma and stressful life events among individuals at clinical high risk for psychosis: a review. Front Psychiatry 8:55. https://doi.org/10. 3389/fpsyt.2017.00055

3. Stanton KJ, Denietolis B, Goodwin BJ, Dvir Y (2020) Childhood trauma and psychosis: an updated review. Child Adolesc Psychiatr Clin N Am 29:115-129. https://doi.org/10.1016/j.chc.2019.08.004

4. Pastore A, de Girolamo G, Tafuri S, Tomasicchio A, Margari F (2020) Traumatic experiences in childhood and adolescence: a meta-analysis of prospective studies assessing risk for psychosis. Eur Child Adolesc Psychiatry. https://doi.org/10.1007/ s00787-020-01574-9

5. Varese F, Smeets F, Drukker M, Lieverse R, Lataster T, Viechtbauer W, Read J, van Os J, Bentall RP (2012) Childhood adversities increase the risk of psychosis: a meta-analysis of patientcontrol, prospective- and cross-sectional cohort studies. Schizophr Bull 38:661-671. https://doi.org/10.1093/schbul/sbs050

6. Pelizza L, Poletti M, Azzali S, Garlassi S, Scazza I, Paterlini F, Chiri LR, Pupo S, Raballo A (2020) Subjective experience of social cognition in adolescents at ultra-high risk of psychosis: findings from a 24-month follow-up study. Eur Child Adolesc Psychiatry 29:1645-1657. https://doi.org/10.1007/ s00787-020-01482-y

7. Bouhaddani SE, van Domburgh L, Schaefer B, Doreleijers TAH, Veling W (2019) Psychotic experiences and trauma predict persistence of psychosocial problems in adolescence. Eur Child Adolesc Psychiatry 28:1597-1606. https://doi.org/10.1007/ s00787-019-01321-9

8. Armando M, Klauser P, Anagnostopoulos D, Hebebrand J, Moreno C, Revet A, Raynaud J-P (2020) Clinical high risk for psychosis model in children and adolescents: a joint position statement of ESCAP Clinical Division and Research Academy. Eur Child Adolesc Psychiatry 29:413-416. https://doi.org/10.1007/ s00787-020-01499-3

9. Poletti M, Pelizza L, Azzali S, Paterlini F, Garlassi S, Scazza I, Chiri LR, Gebhardt E, Pupo S, Andrea R (2019) Clinical high risk for psychosis in childhood and adolescence: findings from the 2-year follow-up of the ReARMS project. Eur Child Adolesc Psychiatry 28:957-971. https://doi.org/10.1007/s00787-018-1262-5

10. Bell IH, Lim MH, Rossell SL, Thomas N (2017) Ecological momentary assessment and intervention in the treatment of psychotic disorders: a systematic review. Psychiatr Serv 68:11721181. https://doi.org/10.1176/appi.ps.201600523 